\title{
Michelle PERROT, Histoire de chambres
}

Paris, Éditions du Seuil, La librairie du XXI ${ }^{\mathrm{e}}$ siècle, 2009, 454 pages

\section{André Burguière}

\section{OpenEdition}

Journals

Édition électronique

URL : https://journals.openedition.org/clio/9944

DOI : $10.4000 /$ clio.9944

ISSN : 1777-5299

Éditeur

Belin

Édition imprimée

Date de publication : 31 décembre 2010

Pagination : 277-280

ISBN : 978-2-8107-0098-1

ISSN : 1252-7017

Référence électronique

André Burguière, « Michelle perrot, Histoire de chambres », Clio. Femmes, Genre, Histoire [En ligne], 32 |

2010, mis en ligne le 31 décembre 2010, consulté le 30 avril 2022. URL : http://

journals.openedition.org/clio/9944; DOI : https://doi.org/10.4000/clio.9944

Ce document a été généré automatiquement le 30 avril 2022.

Tous droits réservés 


\section{Michelle PERROT, Histoire de chambres}

Paris, Éditions du Seuil, La librairie du XXI ${ }^{\mathrm{e}}$ siècle, 2009, 454 pages

\section{André Burguière}

1 «L'ordre de la chambre reproduit l'ordre du monde dont elle est la particule élémentaire", écrit Michelle Perrot en conclusion du bel essai qu'elle vient de consacrer à l'histoire de la chambre comme lieu de vie. Mais son livre dément ou du moins déborde cette proposition trop générale par la complexité sinueuse et aléatoire des transformations dont il reconstitue le cheminement. Dans l'histoire de l'habitat, la chambre individuelle ou conjugale constitue moins une particule élémentaire qu'une pièce rapportée. Pendant longtemps, les sociétés ont voulu ignorer cette formule d'isolement ; la masse des paysans pauvres ont connu jusqu'au $\mathrm{xx}^{\mathrm{e}}$ siècle le régime de la pièce unique, à la fois salle commune de jour et lieu de couchage la nuit. L'artisanat urbain mais aussi l'artisanat rural inséré dans l'output system, associaient lieu de travail et lieu d'habitation dans une même pièce plurifonctionnelle.

2 Les vastes logis eux-mêmes que se faisait construire l'aristocratie, du château fort médiéval au palais de la Renaissance, ne comportaient aucune pièce où l'on put s'isoler. En affirmant que le manoir anglais avait inventé au début du XVII ${ }^{e}$ siècle le corridor qui permet d'accéder à chaque pièce sans avoir à traverser les autres, Lawrence Stone a peut-être trop vite attribué aux élites anglaises un rôle pionnier dans la genèse de l'individualisme. Car si cette invention avait consacré l'apparition d'une sensibilité nouvelle, le besoin de privacy c'est-à-dire d'intimité, qui se serait diffusée progressivement jusqu'au bas de l'échelle sociale, comment expliquer que Virginia Woolf, une femme de la haute société londonienne, habituée à vivre dans des demeures spacieuses, réclame encore, trois siècles après, le droit d'avoir « une chambre à soi »?

3 La chambre individuelle n'est pas née une fois pour toutes d'une innovation dans l'architecture d'intérieur qui aurait rendu les cohabitants plus indépendants les uns des autres. Conquête ou sanction, désirée ou subie, la chambre comme espace de vie soustrait au regard des autres, a été mille fois réinventée. C'est justement parce que Michelle Perrot associe étroitement l'histoire de la chambre à celle de l'intimité et de l'individualité qu'elle ne peut se satisfaire de la vision linéaire et stratifiée du changement social suggérée par Lawrence Stone. Le temps de cette histoire n'a pas la 
continuité intégrative des transformations qui affectent les structures socioéconomiques; celles dont Ernest Labrousse, il y a plus d'un demi-siècle, invitait à dégager et à mesurer la tendance en concentrant l'observation sur un territoire et une tranche chronologique bien délimités. Le temps des usages qui enferment nos façons de vivre, de ressentir et de penser est au contraire répétitif et discontinu. Il innove en se paraphrasant.

4 C'est pour faire ressortir cette particularité que Michelle Perrot a donné à son livre l'allure d'un récit éclaté. La première partie, consacrée à la chambre du Roi, est un clin d'œil à Michel Foucault et à l'évocation du supplice, la forme ancienne du châtiment, qui introduit Surveiller et punir. En faisant de sa chambre à coucher, où il dort seul, une scène ouverte sur le monde qui expose son corps, dès le lever, au regard des autres, le Roi soleil s'accorde, par la réversibilité du devoir de transparence, le droit d'entrer dans le secret des chambres à coucher de tous ses sujets. Cette absorption de toute la société dans la « privance » du roi n'est pas, à proprement parler, un trait d'archaïsme. Elle découle de la monarchie de droit divin conçue par l'absolutisme et s'exprime dans la symbolique solaire que Louis XIV a choisie pour mettre en scène son pouvoir. Elle ne lui survivra guère. Mais ce degré zéro de l'espace privé a été le levier d'une reconquête de l'intimité dans l'ensemble du corps social.

5 L'agonie du mourant a mis plus de temps à s'exclure de la scène publique que le réveil $\mathrm{du}$ monarque. Les très belles pages que Michelle Perrot consacre à la mort de George Sand doivent se comprendre comme un lointain écho au début du livre. Celle qui fut une pionnière du féminisme avant de s'installer vieillissante dans le rôle rassurant de «bonne dame de Nohant ", s'accorde une mort à l'ancienne : la "bonne mort » où la famille entoure le chevet du mourant pour l'aider à préparer son dernier voyage. Mais en laissant le monde des lettres et de la politique venir la saluer sur son lit d'agonie, George Sand accepte d'offrir le spectacle de sa souffrance et de sa dégradation physique comme un souverain du Grand Siècle. Ce n'est qu'une réminiscence. Car l'usage va bientôt s'imposer de mourir à l'hôpital. La veille thérapeutique des médecins va remplacer les prières des proches. Les mourants ont gagné le droit de garder pour eux l'angoisse et les souffrances des derniers instants. Conquête amère de la solitude.

Déclinant la diversité des usages de la chambre et leur histoire selon la classe sociale, la classe d'âge ou le genre avec une minutie d'entomologiste, Michelle Perrot retrouve partout à l'œuvre la même ambivalence: ce qu'on a construit comme refuge de l'intimité peut devenir espace de la solitude. Ce qu'on a désiré comme lieu d'isolement pour se retrouver soi-même peut devenir un lieu d'enfermement.

Dans cette construction historique de l'intimité, tous les itinéraires et surtout tous les acteurs ne se valent pas. On ne s'étonnera pas qu'une grande historienne des femmes qui a contribué plus qu'aucune autre à promouvoir en France la réflexion sur les questions de genre, accorde dans son Histoire de chambres une place privilégiée au rôle des femmes. Mais ce n'est pas par déformation professionnelle que Michèle Perrot a voulu s'attarder ici tout particulièrement sur des parcours féminins. L'association de la femme à l'intimité et à l'intériorité est un trait culturel de longue durée qu'on retrouve dans la plupart des civilisations.

Cette association est problématique. L'épisode du salon des Précieuses tel que le retrace Michelle Perrot, le souligne remarquablement. C'est parce que sa santé fragile l'obligeait à garder la chambre, loin de la Cour, que la marquise de Rambouillet prit l'habitude de convier ses amies à venir converser dans sa «ruelle». C'est en 
transformant leur chambre en salon, en faisant de leur pouvoir sur l'ordre intérieur de la maison le point d'appui d'une critique du monde extérieur et de la sociabilité que les Précieuses ont inventé un nouveau modèle de civilité. Ce modèle a colonisé la Cour et plus tard les classes populaires par le truchement des manuels de civilité puérile en usage dans les « petites écoles » des lasalliens.

Chez les ouvriers français du xIX ${ }^{e}$ siècle, l'assignation des femmes aux valeurs de l'intimité a pris les mêmes formes contradictoires. Ils ont envié le mode de vie bourgeois et son modèle de femme au foyer. Mais ils ont dû attendre longtemps, plus que les ouvriers anglais ou américains, avant de pouvoir offrir à leur "bourgeoise ", le privilège de rester à la maison; et ils ont pu le lui offrir au moment où elle n'en rêvait plus. Les notables paternalistes, promoteurs du « logement social », ont cru également qu'en offrant des logements décents et spacieux aux familles ouvrières, ils renforceraient l'influence civilisatrice de la femme, plus attirée par les joies de l'intimité que par les rencontres du cabaret. En réalité l'habitat social a plus contribué à faire baisser la natalité des familles ouvrières en inspirant aux femmes la détestation des logements surpeuplés qu'à détourner les ouvriers des réunions et des idées subversives.

10 Michelle Perrot montre à quel point ces hypothèses sur les partages sexués des attitudes à l'égard du logement doivent être nuancées. Les ouvriers ont été, au XIX ${ }^{\mathrm{e}}$ siècle, moins indifférents au confort et à l'intimité du foyer qu'on ne l'a dit. Ce qui détermine le confort et la bonne tenue des logements ouvriers, décrits par les enquêteurs leplaysiens dans Les ouvriers des Deux Mondes, tient moins à l'influence de la femme qu'au niveau de revenu du foyer. Les ouvrières ont recherché la privauté d'une chambre séparée pour elles-mêmes avant de la rechercher pour leur ménage. Elles ont fui leurs dortoirs collectifs plus vite que les ouvriers leurs "chambrées ». À tous les niveaux de la société, les femmes ont recherché plus que les hommes un espace privé capable de les rendre à elles-mêmes, pour travailler, pour rêver, pour écrire, pour prier, pour retrouver l'être aimé ou pour retrouver Dieu. Au lieu du retour à soi désiré, elles ont souvent trouvé l'enfermement et la solitude.

11 Cet essai est composé comme une succession de scènes qui s'enchaînent si naturellement qu'on ne peut résister à l'envie de lire le livre d'une seule traite, comme un roman. Ce n'est pourtant ni un récit romanesque bien que l'auteure, abandonnant la vieille méfiance de l'histoire des groupes et des structures à l'égard des témoignages littéraires, emprunte au roman une part non négligeable de sa documentation, ni un récit historique si l'on entend par là le parcours d'une institution dont l'état présent représenterait l'aboutissement. Les chambres dans lesquelles Michelle Perrot nous introduit en poussant l'une après l'autre les portes qui protégeaient leur intimité, ne composent pas pour autant un musée imaginaire de l'espace privé. Elles proposent une histoire. Mais laquelle?

La démarche historienne qu'a choisie Michelle Perrot pour cet essai en privilégie, dans ses analyses, le témoignage des acteurs eux-mêmes et des romans qui ont su transcrire dans la fiction le vécu de leur époque.

Il y a quelque chose de mémoriel, au sens proustien du terme, dans cet effort pour retrouver sous les souvenirs des acteurs du passé, la trace des émotions et des idées qui ont été englouties par le flot du temps. Mais cette démarche mémorielle ne se confond pas avec la sacralisation de la mémoire collective comme actualisation du passé utile qui déferle aujourd'hui. Faute de pouvoir désormais attribuer au mouvement de 
l'histoire une signification d'ensemble permettant de distinguer dans le passé le dessin d'un avenir, l'habitude s'installe de ne donner sens qu'à ce qui du passé oblige la mémoire, c'est-à-dire à ce qui nous permet de nous sentir victimes ou coupables.

14 Le passé que réveille Michelle Perrot par la reconstitution d'une multitude d'expériences personnelles ordinaires qui ont inventé ou transformé nos usages de l'espace privé, ne sert pas à nourrir les énoncés de la mémoire collective par lesquels nous recherchons l'accord avec les autres et le consentement à l'ordre du monde. Extraite de la masse ensevelie de la mémoire inconsciente, cette multitude de fils rouges que tire l'historienne pour mettre à jour leur cheminement et l'intrication, nous aide à comprendre ce que nous sommes à travers les habitudes qui nous ont formés et à en admettre la contingence. 